\title{
MAGNUS CARLEHED
}

\section{Potentials in pluripotential theory}

Annales de la faculté des sciences de Toulouse $6^{e}$ série, tome $8, \mathrm{n}^{\circ} 3$ (1999), p. 439-469

<http://www.numdam.org/item?id=AFST_1999_6_8_3_439_0>

(C) Université Paul Sabatier, 1999, tous droits réservés.

L'accès aux archives de la revue «Annales de la faculté des sciences de Toulouse » (http://picard.ups-tlse.fr/ annales/) implique l'accord avec les conditions générales d'utilisation (http://www.numdam.org/conditions). Toute utilisation commerciale ou impression systématique est constitutive d'une infraction pénale. Toute copie ou impression de ce fichier doit contenir la présente mention de copyright.

\section{Numdam}

Article numérisé dans le cadre du programme Numérisation de documents anciens mathématiques http://www.numdam.org/ 


\title{
Potentials in pluripotential theory ${ }^{(*)}$
}

\author{
MAGNUS CARLEHED ${ }^{(1)}$
}

RÉSuMÉ. - Nous traitons des potentiels des mesures positives dans un domaine de $\mathbb{C}^{n}$, où le noyau est le logarithme ou la fonction de Green pluricomplexe. Leur mesure de Monge-Ampère est calculée, et nous étudions quelles propriétés des potentiels classiques demeurent.

Abstract. - We discuss potentials of positive measures in domains in $\mathbb{C}^{n}$, where the kernel is the logarithm or the pluricomplex Green function. Their Monge-Ampère measure is computed, and we investigate which properties of classical potentials remain.

\section{Introduction and definition of potentials}

In classical potential theory in $\mathbb{R}^{n}$, potentials of positive measures play a crucial role. The theory is linear and, due to Riesz decomposition theorem, subharmonic functions are essentially potentials modulo harmonic functions. Furthermore, we can solve Dirichlet problems using convolutions. In the case $n=2$ we can equally well work in the complex plane, and the Laplacian is invariant under conformal mappings. For a thorough treatment of classical potential theory we refer to the book by Hayman and Kennedy [Ha-Ke]. However, from the point of view of complex analysis classical potential theory is unsatisfactory. The Laplacian is not biholomorphically invariant in higher dimensions.

(*) Reçu le 19 juin 1998, accepté le 12 octobre 1999

(1) Linköping University, ITN, Campus Norrköping, 60174 Norrköping, Sweden. E-mail address: magca@itn.liu.se 
Pluripotential theory is the study of plurisubharmonic functions and the complex Monge-Ampère operator. The operator is defined on certain classes of plurisubharmonic functions in domains in $\mathbb{C}^{n}$. In the case $n=1$, it reduces to the Laplacian, but in higher dimension it is non-linear. It is invariant under biholomorphic mappings. We refer to Klimek's monograph $[\mathrm{Kl}]$ for an introduction to pluripotential theory and the basic definitions.

Since pluripotential theory is non-linear, we can not expect potentials to be as fruitful as in the classical case. Still, it makes sense to study them, and ask which properties remain, which is the purpose of this article.

We recall the following definition of the multipole Green function.

Definition 1.1 [Lel2]. - Let $\Omega$ be a domain in $\mathbb{C}^{n}$, and let

$$
A=\left\{\left(a_{k}, \nu_{k}\right), 1 \leqslant k \leqslant p\right\}
$$

be a finite system of points $a_{k} \in \Omega$ with weights $\nu_{k}>0$. Define

$$
g(z, A)=\sup \{v(z): v \in U(A), v<0\}
$$

where

$$
U(A)=\left\{v \in P S H(\Omega): v(\zeta) \leqslant \nu_{k} \log \left|\zeta-a_{k}\right|+O(1), \zeta \rightarrow a_{k}, 1 \leqslant k \leqslant p\right\} .
$$

We call this the pluricomplex Green function for $\Omega$, relative to the system $A$.

In hyperconvex domains the Green function is continuous, and tends to zero at the boundary [Lel2]. In the case $p=1, a_{1}=w, \nu_{1}=1$ we have the standard pluricomplex Green function $g(z, w)$ with one pole at $w \in \Omega$.

The following theorem shows that it makes sense to talk about potentials in pluripotential theory.

ThEOREM 1.2 [Lel1]. - Let $\Omega$ be a domain in $\mathbb{C}^{n},(T, \mu)$ a locally compact measure space, with a positive measure $\mu$. Let $K: \Omega \times T \rightarrow[-\infty, \infty)$ be a function such that

1) $K\left(z e^{i \theta}, t\right)$ is measurable with respect to the product measure $d \theta \otimes \mu$,

2) $z \mapsto K(z, t)$ is plurisubharmonic for each $t \in T$,

3) $t \mapsto K(z, t)$ is measurable for each $z \in \Omega$,

4) the function $z \mapsto \sup _{t \in T} K(z, t)$ is locally upper bounded on $\Omega$.

Then $q_{\mu}^{K}(z):=\int_{T} K(z, w) d \mu(w)$ is plurisubharmonic on $\Omega$.

We will mainly study two special cases, so we introduce special notation for these. 


\section{Potentials in pluripotential theory}

DEFINITION 1.3. - Let $\mu$ be a finite, positive measure with support in $\Omega$, where $\Omega$ is a bounded domain in $\mathbb{C}^{n}$. Let $g(z, w)$ be the pluricomplex Green function for $\Omega$ with pole in $w \in \Omega$. We define the pluricomplex potential of $\mu$ as

$$
p_{\mu}(z)=\int_{\Omega} g(z, w) d \mu(w),
$$

and the logarithmic potential of $\mu$ as

$$
l p_{\mu}(z)=\int_{\Omega} \log |z-w| d \mu(w) .
$$

Clearly both potentials are plurisubharmonic in $\Omega$, and if $\Omega$ is hyperconvex, then $p_{\mu}$ vanishes on the boundary. For the logarithmic potential in $\mathbb{R}^{2 n}$, we refer to [Ha-Ke], Chapter 5 .

In Section 2 we prove a characterization of logarithmic potentials, which can be viewed as a counterpart to Riesz decomposition theorem.

In Section 3 we give some examples of pluricomplex potentials in the unit ball.

It is possible to define the measures

$$
d d^{c} g\left(z, v_{1}\right) \wedge \ldots \wedge d d^{c} g\left(z, v_{n}\right)
$$

and

$$
d d^{c} \log \left|z-v_{1}\right| \wedge \ldots \wedge d d^{c} \log \left|z-v_{n}\right| .
$$

This is done in Section 4, and there we also show that the latter is absolutely continuous with respect to the Lebesgue measure unless all $v_{i}$ coincide. If $g(\cdot, v) \in C^{2}(\Omega \backslash\{v\})$ for all $v \in \Omega$, the same holds for $d d^{c} g\left(z, v_{1}\right) \wedge \ldots \wedge$ $d d^{c} g\left(z, v_{n}\right)$.

Then (Section 5) we extend the comparison principle and partial integration formulas to certain classes of unbounded plurisubharmonic functions.

In Section 6, we define so-called integrated measures. Typically we will have a family of positive measures $\nu\left(v_{1}, \ldots, v_{n}\right)$, depending on the parameters $v_{1}, \ldots, v_{n}$, such that the total mass is bounded, uniformly in all the parameters. Examples are (1) and (2). We then define measures

$$
\int_{\Omega^{k}} \nu\left(v_{1}, \ldots, v_{n}\right) d \mu\left(v_{1}\right) \cdots d \mu\left(v_{k}\right),
$$

where $1 \leqslant k \leqslant n$, and $\mu$ is a given positive measure. 
After that we prove representation formulas for the Monge-Ampère measures of bounded potentials. For the pluricomplex potential, the formula is

$$
\left(d d^{c} p_{\mu}(z)\right)^{n}=\int_{\Omega^{n}} d d_{z}^{c} g\left(z, v_{1}\right) \wedge \ldots \wedge d d_{z}^{c} g\left(z, v_{n}\right) d \mu\left(v_{1}\right) \cdots d \mu\left(v_{n}\right) .
$$

This is done in Section 7.

In Section 8, we perform the procedure $\mu \mapsto p_{\mu} \mapsto\left(d d^{c} p_{\mu}\right)^{n}$. It turns out that if $g(\cdot, v) \in C^{2}$ and $p_{\mu}$ is bounded, then t...e procedure is "smoothing", i.e. the resulting measure is absolutely continuous, even if $\mu$ is not. We also prove some estimates for this procedure.

Finally, in Section 9, we investigate measures whose pluricomplex potentials in the unit ball have finite energy in the sense of Cegrell [Ce1]. We give a sufficient condition for this, and extend the representation formula to that class. The procedure $\mu \mapsto p_{\mu} \mapsto\left(d d^{c} p_{\mu}\right)^{n}$ is still "smoothing". We also give an example of a measure such that the potential is unbounded but of finite energy.

We would like to mention two other motivations to study potentials in several complex variables. The first is the concept of pluri-thin sets. For a discussion of these, we refer the reader to [Ca]. The second motivation is the following. We define $C$ to be the cone of non-positive plurisubharmonic functions in a fixed hyperconvex domain. We say that a function $\varphi$ in $C$ is extremal, if $\varphi=\varphi_{1}+\varphi_{2}$, where each $\varphi_{i}$ is in $C$, implies that there are positive constants $\alpha_{i}$ such that $\varphi_{i}=\alpha_{i} \varphi, i=1,2$. It is known that the pluricomplex Green function with one pole is always extremal [Ce-T]. From the general Choquet theory of convexity the following theorem is known. Let $C$ be a convex cone. Then for each $q \in C$ there is a positive measure $\mu_{q}$ supported on the extreme elements of $C$ such that $f(q)=\int f(x) d \mu_{q}(x)$ for all affine functions $f$. In our situation, taking $f$ as a point evaluation at a point $a$ in the domain, we find that for any function $\varphi \in C$ there is a measure $\mu_{\varphi}$ such that $\varphi(a)=\int x(a) d \mu_{\varphi}(x)$ for all $a$ in the domain. Here $x$ varies over all extremal plurisubharmonic functions. A pluricomplex potential is a special case of this, namely where the measure is supported only on the (one-polar) pluricomplex Green functions. We do not carry this discussion any further in this paper, however, the results indicate that the pluricomplex potentials form a very small subset of $C$ (see Theorem 8.1). Therefore there should be plenty of extremal plurisubharmonic functions, in addition to the Green functions. To characterize all of these seems almost impossible, but it would be interesting to have at least a few more examples.

We also make a general remark about dimension. Most of the results of this paper are valid for domains in $\mathbb{C}^{n}$, but to avoid too unwieldy expressions 


\section{Potentials in pluripotential theory}

and computations, some results in Sections 8 and 9 are done in $\mathbb{C}^{2}$ only. However, we suspect that they are true in higher dimensions too.

Most of the results of this article can be found in Paper I in [Ca], and in some cases more details are given there.

Acknowledgements. I thank my thesis advisor Urban Cegrell for his guidance and support and the referee for helpful suggestions. Part of this research was carried out while I was visiting Department of Mathematics and Statistics, University of Canterbury, Christchurch, New Zealand, and I thank the staff there for their hospitality. This work was partly supported by Magnuson's foundation (Royal Swedish Academy of Sciences) and Lars Hierta memorial foundation.

\section{Characterization of logarithmic potentials}

For logarithmic potentials, it is possible to give a counterpart to the Riesz decomposition theorem.

Proposition 2.1. - There is a positive constant $C_{n}$, such that $\Delta^{n} l p_{\mu}=$ $C_{n} \mu$, in the distributional sense, for each measure $\mu$. Conversely, if $u$ is a real-valued function, such that $\Delta^{n} u$ exists in the distributional sense, and equals a positive measure $C_{n} \mu$, then locally we have

$$
u=l p_{\mu}+b
$$

where $b$ is a real analytic function, and $\triangle^{n} b=0$.

Proof. - The first claim follows from the fact that $\log |x|$ is a fundamental solution for the operator $\Delta^{n}$ in $\mathbb{R}^{2 n}$, see [A-Cr-Li]. To prove the second, note that

$$
\triangle^{n}\left(u-l p_{\mu}\right)=0
$$

in the sense of distributions. Since the operator $\Delta^{n}$ is elliptic it has only classical solutions, and hence $u-l p_{\mu}$ is an $n$-harmonic function. These are real analytic.

Remark. - In a relatively compact subset of $\Omega$, which is star-shaped with centre $a$, we can write $b$ as

$$
b(z)=\sum_{j=0}^{n-1}|z-a|^{2 j} h_{j}(z),
$$

where each $h_{j}$ is harmonic. This is called the Almansi expansion [A-Cr-Li]. 


\section{Some explicit examples of pluricomplex potentials in the unit ball}

In $[\mathrm{Ca}]$ the pluricomplex potentials of some measures are computed explicitly. Here we only state the results, and refer the reader to [Ca] for the proofs.

Proposition 3.1. - Let $B$ be the unit ball in $\mathbb{C}^{2}$, and $\mu$ be the normalized Lebesgue measure on the disc $D \times\{0\}$. Then

$$
p_{\mu}(z)=\frac{\left(|z|^{2}-1\right)\left(1-\left|z_{2}\right|^{2}+\left|z_{2}\right|^{2} \log \left|z_{2}\right|^{2}\right)}{2\left(1-\left|z_{2}\right|^{2}\right)^{2}}
$$

Proposition 3.2. - Let $\sigma=\sigma_{r}$ be the surface measure on the sphere $\partial B(0, r)$ in $\mathbb{C}^{2}, r>0$. Let $t=|z|$. Then

$p_{\sigma}(z)=p_{\sigma_{r}}(z)=\pi^{2} r^{3} \cdot\left\{\begin{array}{l}\log r^{2}+\frac{1-r^{2}}{r^{2}}+\frac{\left(1-r^{2}\right)\left(1-t^{2}\right)}{r^{2} t^{2}} \log \left(1-t^{2}\right), \text { if } t \leqslant r \\ \log t^{2}+\frac{1-t^{2}}{t^{2}}+\frac{\left(1-r^{2}\right)\left(1-t^{2}\right)}{r^{2} t^{2}} \log \left(1-r^{2}\right), \text { if } t \geqslant r\end{array}\right.$

(To get the potential of the normalized surface measure, divide by $2 \pi^{2} r^{3}$.)

THEOREM 3.3. - Let $\mu$ be the normalized Lebesgue measure in the unit ball, i.e. $\lambda / b_{2 n}$, where $\lambda$ is the Lebesgue measure. Then the potential is

$$
p_{\mu}(z)=\left(|z|^{2}-1\right) /(2 n)
$$

and hence

$$
\left(d d^{c} p_{\mu}(z)\right)^{n}=\left(\frac{2 \pi}{n}\right)^{n} \mu
$$

\section{Definition of the Monge-Ampère operator on certain classes of unbounded functions}

It is possible to define measures of the type $d d^{c} u^{1} \wedge \ldots \wedge d d^{c} u^{n}$, where each $u^{j}$ is plurisubharmonic and locally bounded outside a finite set $A$. This can be done by generalizing a method used by Klimek $[\mathrm{Kl}]$ in the case where $A$ consists of only one point. We refer the reader to [Kl] and [Ca] for details. The construction is only a special case of the much more general treatment in $[\mathrm{S}]$. 
Let $\Omega$ be a domain in $\mathbb{C}^{n}$, and $A=\left\{a_{1}, \ldots, a_{p}\right\}$ a fixed finite subset of $\Omega$. Define

$$
P S H(\Omega ; A)=P S H(\Omega) \cap L_{l o c}^{\infty}(\Omega \backslash A) .
$$

Proposition 4.1. - Let $u^{1}, \ldots, u^{n}$ be functions in $P S H(\Omega ; A)$. Then there exists a positive Borel measure $\mu$ on $\Omega$ such that if $\left\{u_{i}^{j}\right\}_{1 \leqslant i, 1 \leqslant j \leqslant n} \subset$ $P S H \cap L_{\text {loc }}^{\infty}(\Omega)$ and $u_{i}^{j} \searrow u^{j}$, when $i \rightarrow \infty$, for all $1 \leqslant j \leqslant n$, then $d d^{c} u_{i}^{1} \wedge$ $\ldots \wedge d d^{c} u_{i}^{n}$ is weak ${ }^{*}$-convergent to $\mu$.

Definition 4.2. - We define $d d^{c} u^{1} \wedge \ldots \wedge d d^{c} u^{n}=\mu$, where $\mu$ is the measure of the preceding proposition.

The special cases we have in mind are

$$
d d^{c} g\left(z, v_{1}\right) \wedge \ldots \wedge d d^{c} g\left(z, v_{n}\right)
$$

and

$$
d d^{c} \log \left|z-v_{1}\right| \wedge \ldots \wedge d d^{c} \log \left|z-v_{n}\right| .
$$

In these expressions, we always allow points to be repeated. It is well-known (see e.g. [Kl]) that if all the $v_{j}$ coincide, and are equal to $v$, then both these measures are equal to $(2 \pi)^{n} \delta_{v}$. We now want to examine what happens if they do not coincide. We will need the following special case of a theorem by Demailly [D].

Theorem 4.3. - Assume that $a \in \Omega, u, v \in P S H \cap C(\Omega,[-\infty, \infty[)$, $u<v$ in $\Omega \backslash\{a\}, u^{-1}(\{-\infty\})=v^{-1}(\{-\infty\})=\{a\}$, and

$$
\varlimsup_{z \rightarrow a} \frac{u(z)}{v(z)}=1 .
$$

Then $\left(d d^{c} u\right)^{n}(\{a\}) \leqslant\left(d d^{c} v\right)^{n}(\{a\})$. If the upper limit is a limit, then equality holds.

Proposition 4.4. - Assume that $v_{j} \in \Omega$, for all $j$, and that $v_{i} \neq v_{j}$ for some indices $i$ and $j$. Then $d d^{c} \log \left|z-v_{1}\right| \wedge \ldots \wedge d d^{c} \log \left|z-v_{n}\right|$ is an absolutely continuous measure with respect to the Lebesgue measure. If $g(\cdot, v) \in C^{2}(\Omega \backslash\{v\})$ for all $v \in \Omega$, then $d d^{c} g\left(z, v_{1}\right) \wedge \ldots \wedge d d^{c} g\left(z, v_{n}\right)$ is an absolutely continuous measure with respect to the Lebesgue measure.

Proof. - We prove the proposition for $g$, the proof for the logarithm being essentially the same. Let $1 \leqslant k \leqslant n-1$. It is sufficient to show that if $b_{j} \neq a$, for all $1 \leqslant j \leqslant n-k$, then

$$
\int_{\{a\}}\left(d d^{c} g(z, a)\right)^{k} \wedge d d^{c} g\left(z, b_{1}\right) \wedge \ldots \wedge d d^{c} g\left(z, b_{n-k}\right)=0 .
$$


Define

$$
u(z)=g(z, a)+\sum_{j=1}^{n-k} g\left(z, b_{j}\right) .
$$

Then using Theorem 4.3 near $a$, we obtain $\int_{\{a\}}\left(d d^{c} u\right)^{n}=\int_{\{a\}}\left(d d^{c} g(z, a)\right)^{n}=$ $(2 \pi)^{n}$. On the other hand, expanding the wedge product, we get

$$
\begin{aligned}
\left(d d^{c} u\right)^{n}= & \left(d d^{c} g(z, a)\right)^{n}+\left(d d^{c} g(z, a)\right)^{k} \wedge d d^{c} g\left(z, b_{1}\right) \wedge \ldots \wedge d d^{c} g\left(z, b_{n-k}\right) \\
& + \text { other terms } \\
\geqslant & (2 \pi)^{n} \delta_{a}+\left(d d^{c} g(z, a)\right)^{k} \wedge d d^{c} g\left(z, b_{1}\right) \wedge \ldots \wedge d d^{c} g\left(z, b_{n-k}\right) .
\end{aligned}
$$

Thus

$$
\begin{aligned}
\int_{\{a\}} & \left(d d^{c} g(z, a)\right)^{k} \wedge d d^{c} g\left(z, b_{1}\right) \wedge \ldots \wedge d d^{c} g\left(z, b_{n-k}\right) \\
& \leqslant \int_{\{a\}}\left(d d^{c} u\right)^{n}-(2 \pi)^{n}=0
\end{aligned}
$$

and the proof is complete.

The hypothesis about $g$ being $C^{2}$ is fulfilled in a strictly convex domain with smooth boundary [Lem].

Let $A$ be as in Definition 1.1. Define

$$
h(z, A)=\sum_{j=1}^{p} \nu_{j} g\left(z, a_{j}\right)
$$

As an example, let us assume that $\Omega \subset \mathbb{C}^{2}$ is such that $g \in C^{2}$, and compute $\left(d d^{c} h(z, A)\right)^{2}$ :

$$
\begin{aligned}
\left(d d^{c} h(z, A)\right)^{2} & =\sum_{j} \nu_{j}^{2}\left(d d^{c} g\left(z, a_{j}\right)\right)^{2}+\sum_{j \neq k} \nu_{j} \nu_{k} d d^{c} g\left(z, a_{j}\right) \wedge d d^{c} g\left(z, a_{k}\right) \\
& =(2 \pi)^{2} \sum_{j} \nu_{j}^{2} \delta_{a_{j}}+\sum_{j \neq k} \nu_{j} \nu_{k} d d^{c} g\left(z, a_{j}\right) \wedge d d^{c} g\left(z, a_{k}\right) .
\end{aligned}
$$

The right hand side is the Lebesgue decomposition of the measure relative to the Lebesgue measure, and the first term of the right hand side is $\left(d d^{c} g(z, A)\right)^{2}$.

\section{Comparison principle and partial integration for certain unbounded plurisubharmonic functions}

In what follows we will often need to compare certain measures and do partial integration. We therefore need to justify this in a more general setting than the standard one. 
Definition 5.1. - For any function $u$ with values in $[-\infty, \infty)$ and any integer $L$, define $u^{L}(z)=\max \{u(z), L\}$.

Proposition 5.2. - Let $\Omega$ be a bounded domain in $\mathbb{C}^{n}$, and suppose that $\varphi_{1}, \ldots, \varphi_{n}$ are plurisubharmonic functions in $\Omega$. Assume that for some neighbourhood $U$ of $\partial \Omega$, all $\varphi_{i}$ are bounded in $U \cap \Omega$. Set

$$
L=\min _{i=1, \ldots, n}\left(\inf _{z \in U \cap \Omega} \varphi_{i}(z)\right) .
$$

Then, for all $M, N \leqslant L$,

$$
\int_{\Omega} d d^{c} \varphi_{1}^{M} \wedge \ldots \wedge d d^{c} \varphi_{n}^{M}=\int_{\Omega} d d^{c} \varphi_{1}^{N} \wedge \ldots \wedge d d^{c} \varphi_{n}^{N} .
$$

We omit the simple proof, which basically depends on the fact that the functions coincide in a neighbourhood of the boundary. See [Ca] for details.

Corollary 5.3. - With the same assumptions as in Proposition 5.2, if

$$
\int_{\Omega} d d^{c} \varphi_{1} \wedge \ldots \wedge d d^{c} \varphi_{n}
$$

is defined, it equals

$$
\int_{\Omega} d d^{c} \varphi_{1}^{M} \wedge \ldots \wedge d d^{c} \varphi_{n}^{M}
$$

for any $M$.

COROLlaRY 5.4 (Comparison principle). - Let $\Omega$ be a hyperconvex, bounded domain in $\mathbb{C}^{n}$. Let $u$ and $v$ be plurisubharmonic functions on $\Omega$, and suppose that $u \leqslant v$. Further assume that $u$ and $v$ are upper bounded exhaustions, that is $\{z \in \Omega: u<c\} \subset \subset \Omega$ for all $c<0$, and similarly for $v$. Then

$$
\int_{\Omega}\left(d d^{c} u\right)^{n} \geqslant \int_{\Omega}\left(d d^{c} v\right)^{n}
$$

Proof. - For bounded functions, this is Lemma V:3 in [Ce3]. Hence we have

$$
\int_{\Omega}\left(d d^{c} u^{M}\right)^{n} \geqslant \int_{\Omega}\left(d d^{c} v^{M}\right)^{n}
$$

for all $M \leqslant-1$. Now Proposition 5.2 applies, and the corollary is proved. $\square$

TheOREM 5.5 (Partial integration). - Let $\Omega$ be a hyperconvex, bounded domain in $\mathbb{C}^{n}$. Let $\varphi_{0}, \varphi_{1}, \ldots, \varphi_{n}$ be plurisubharmonic functions in $\Omega$, and 
assume that for some neighbourhood $U$ of $\partial \Omega$, all $\varphi_{i}$ are bounded in $U \cap \Omega$. Further assume that $\varphi_{0}$ and $\varphi_{1}$ are exhaustions, continuous as functions $\Omega \rightarrow[-\infty, 0)$, and that

$$
\int d d^{c} \varphi_{1} \wedge \ldots \wedge d d^{c} \varphi_{n}+\int d d^{c} \varphi_{0} \wedge d d^{c} \varphi_{2} \wedge \ldots \wedge d d^{c} \varphi_{n}<\infty
$$

Then partial integration is allowed, that is

$$
\int_{\Omega} \varphi_{0} d d^{c} \varphi_{1} \wedge \ldots \wedge d d^{c} \varphi_{n}=\int_{\Omega} \varphi_{1} d d^{c} \varphi_{0} \wedge d d^{c} \varphi_{2} \wedge \ldots \wedge d d^{c} \varphi_{n}
$$

We remark that the theorem applies to the class of functions we studied in Section 4, but is actually more general. We refer to [S] for the definition of the Monge-Ampère operator in the more general case.

For the proof of this theorem we refer the reader to the following sources: [B12], [Ce4] (where an even more general result is proved) or [Ca].

\section{Definition of integrated measures}

Let $\Omega$ be a hyperconvex, bounded domain in $\mathbb{C}^{n}$, and suppose that $A$ is a finite subset of $\Omega$. We refer to Section 1 for notation and the definition of the multipole pluricomplex Green function. Here we will make the following convention. Let $B=\left(v_{1}, \ldots, v_{n}\right)$ be an $n$-tuple of points in $A$, where some $v_{j}$ might coincide. Let $\widetilde{B}=\left\{v_{1}, \ldots, v_{n}\right\}=\left\{v_{j_{1}}, \ldots, v_{j_{p}}\right\}$, where all $v_{j_{k}}$ are different. Set $D=\left(v_{j_{k}}, 1\right)_{k=1}^{p}$. Abusing notation, we will write $g(z, B)$ instead of $g(z, D)$.

Note that if $v \in \widetilde{B}$, we have

$$
0>g(z, v) \geqslant g(z, B)
$$

for all $z \in \Omega \backslash\{v\}$. Hence

$$
\sum_{j=1}^{n} g\left(z, v_{j}\right) \geqslant n g(z, B),
$$

for all $z \in \Omega \backslash A$. Corollary 5.4 gives

$$
\begin{aligned}
& \int_{\Omega} d d_{z}^{c} g\left(z, v_{1}\right) \wedge \ldots \wedge d d_{z}^{c} g\left(z, v_{n}\right) \\
& \leqslant \int_{\Omega}\left(d d_{z}^{c} \sum_{j=1}^{n} g\left(z, v_{j}\right)\right)^{n} \leqslant \int_{\Omega}\left(d d_{z}^{c} n g(z, B)\right)^{n}=p(2 \pi n)^{n} \leqslant n(2 \pi n)^{n} . \\
& -448-
\end{aligned}
$$


We conclude that the total mass of the measures $d d_{z}^{c} g\left(z, v_{1}\right) \wedge \ldots \wedge$ $d d_{z}^{c} g\left(z, v_{n}\right)$ is bounded, uniformly in $\left(v_{1}, \ldots, v_{n}\right) \in \Omega^{n}$. Let $\mu$ be a finite measure on $\Omega$. Using this fact, we can make the following definition.

DEFINITION 6.1. - For each $1 \leqslant k \leqslant n$, we define a new positive measure

$$
\int_{\Omega^{k}} d d_{z}^{c} g\left(z, v_{1}\right) \wedge \ldots \wedge d d_{z}^{c} g\left(z, v_{n}\right) d \mu\left(v_{1}\right) \cdots d \mu\left(v_{k}\right)
$$

in the following way:

$$
\begin{aligned}
& \int_{\Omega} \chi(z)\left(\int_{\Omega^{k}} d d_{z}^{c} g\left(z, v_{1}\right) \wedge \ldots \wedge d d_{z}^{c} g\left(z, v_{n}\right) d \mu\left(v_{1}\right) \cdots d \mu\left(v_{k}\right)\right) \\
& :=\int_{\Omega^{k}}\left(\int_{z \in \Omega} \chi(z) d d_{z}^{c} g\left(z, v_{1}\right) \wedge \ldots \wedge d d_{z}^{c} g\left(z, v_{n}\right)\right) d \mu\left(v_{1}\right) \cdots d \mu\left(v_{k}\right),
\end{aligned}
$$

where $\chi \in C_{0}^{\infty}(\Omega)$.

Note that the measure is a function in the variables $v_{k+1}, \ldots, v_{n}$.

Now, let $\Omega$ be any bounded domain in $\mathbb{C}^{n}$. Let $K(z, w)$ be defined as in Theorem 1.2, and suppose that $K$ is bounded on $\Omega \times T$. In an analogous manner to the above definition, we also define the following measures:

$$
\begin{aligned}
& \int_{\Omega} \chi(z)\left(\int_{\Omega^{k}} d d_{z}^{c} \log \left|z-v_{1}\right| \wedge \ldots \wedge d d_{z}^{c} \log \left|z-v_{k}\right| d \mu\left(v_{1}\right) \cdots d \mu\left(v_{k}\right)\right) \\
& :=\int_{\Omega^{k}}\left(\int_{z \in \Omega} \chi(z) d d_{z}^{c} \log \left|z-v_{1}\right| \wedge \ldots \wedge d d_{z}^{c} \log \left|z-v_{k}\right|\right) d \mu\left(v_{1}\right) \cdots d \mu\left(v_{k}\right),
\end{aligned}
$$

and

$$
\begin{aligned}
& \int_{\Omega} \chi(z)\left(\int_{T^{k}} d d_{z}^{c} K\left(z, v_{1}\right) \wedge \ldots \wedge d d_{z}^{c} K\left(z, v_{n}\right) d \mu\left(v_{1}\right) \cdots d \mu\left(v_{k}\right)\right) \\
& :=\int_{T^{k}}\left(\int_{z \in \Omega} \chi(z) d d_{z}^{c} K\left(z, v_{1}\right) \wedge \ldots \wedge d d_{z}^{c} K\left(z, v_{n}\right)\right) d \mu\left(v_{1}\right) \cdots d \mu\left(v_{k}\right),
\end{aligned}
$$

where $\chi \in C_{0}^{\infty}(\Omega)$.

\section{A formula for the Monge-Ampère measures of potentials}

The goal of this section is to prove representation formulas for the Monge-Ampère measure of potentials. In fact the following is true. 
Proposition 7.1. - Let $\Omega$ be a bounded domain in $\mathbb{C}^{n}$, and $\mu$ a finite measure. Let $K(z, w)$ and $q_{\mu}^{K}$ be defined as in Theorem 1.2, and suppose that $K$ is bounded on $\Omega \times T$. Then we have

$$
\left(d d^{c} q_{\mu}^{K}(z)\right)^{n}=\int_{T^{n}} d d_{z}^{c} K\left(z, v_{1}\right) \wedge \ldots \wedge d d_{z}^{c} K\left(z, v_{n}\right) d \mu\left(v_{1}\right) \cdots d \mu\left(v_{n}\right) .
$$

Further assume that $\mu$ is such that $l p_{\mu}$ is bounded. Then the formula

$$
\left(d d^{c} l p_{\mu}(z)\right)^{n}=\int_{\Omega^{n}} d d_{z}^{c} \log \left|z-v_{1}\right| \wedge \ldots \wedge d d_{z}^{c} \log \left|z-v_{n}\right| d \mu\left(v_{1}\right) \cdots d \mu\left(v_{n}\right)
$$

holds. Finally, if $\Omega$ is hyperconvex, and $\mu$ is such that $p_{\mu}$ is bounded, we have

$$
\left(d d^{c} p_{\mu}(z)\right)^{n}=\int_{\Omega^{n}} d d_{z}^{c} g\left(z, v_{1}\right) \wedge \ldots \wedge d d_{z}^{c} g\left(z, v_{n}\right) d \mu\left(v_{1}\right) \cdots d \mu\left(v_{n}\right) .
$$

The three formulas are equalities between positive measures in the variable $z$.

To prove these formulas we need the following lemmas.

LEMMA 7.2 (Chern-Levine-Nirenberg's inequality). - Let $\Omega$ be an open neighbourhood of a compact set $K \subset \mathbb{C}^{n}$. There exist a constant $C>0$ and a compact set $L \subset \Omega \backslash K$, which depend on $K$ and $\Omega$, such that for all $u_{1}, \ldots, u_{n} \in P S H \cap L^{\infty}(\Omega)$,

$$
\int_{K} d d^{c} u_{1} \wedge \ldots \wedge d d^{c} u_{n} \leqslant C\left\|u_{1}\right\|_{L} \cdots\left\|u_{n}\right\|_{L}
$$

Proof. - We refer to [Kl], Proposition 3.4.2 and Corollary 3.4.8.

Remark. - For later reference, we would like to point out two facts about the proof of Lemma 7.2. Firstly, the inequality is proved by a recursive procedure, and if this is stopped after, say, $n-k$ steps we obtain

$$
\int_{K} d d^{c} u_{1} \wedge \ldots \wedge d d^{c} u_{n} \leqslant C \prod_{j=k+1}^{n}\left\|u_{j}\right\|_{L} \int_{K} d d^{c} u_{1} \wedge \ldots \wedge d d^{c} u_{k} \wedge \beta^{n-k}
$$

where

$$
\beta=\frac{i}{2} \sum_{j=1}^{n} d z_{j} \wedge d \bar{z}_{j}
$$

is the standard Kähler form. Note that $d d^{c}\left(|z|^{2} / 4\right)=\beta$. Secondly, the set $L$ can be chosen "far from" $K$, i.e. for any compact subset $K^{\prime}$ of $\Omega$ such that $K \subset K^{\prime}$, the set $L$ can be chosen in the complement of $K^{\prime}$. However the constant $C$ may change if $L$ changes. 
LEMMA 7.3. - For all $C^{2}$ functions $u_{1}, \ldots, u_{k}$ and all smooth $(n-k, n-$ k) test forms $\phi$ we have

$$
\int_{\Omega} d d^{c} u_{1} \wedge \cdots \wedge d d^{c} u_{k} \wedge \phi=\int_{\Omega} u_{k} d d^{c} u_{1} \wedge \cdots \wedge d d^{c} u_{k-1} \wedge d d^{c} \phi
$$

Proof. - See [Kl], Prop. 3.4.1.

Proof of Proposition 7.1. - We first prove Formula (6). Since $p_{\mu}$ is plurisubharmonic and locally bounded, $\left(d d^{c} p_{\mu}(z)\right)^{n}$ is defined. For each $u \in \Omega$, let $g_{\epsilon}(\cdot, u)$ be the usual regularization of $g(\cdot, u)$, defined on

$$
\Omega_{\epsilon}:=\{z \in \Omega: \operatorname{dist}(z, \partial \Omega)>\epsilon\} .
$$

Then $\left\{g_{\epsilon}(z, u)\right\}$ is a family of negative, $C^{\infty}$ functions defined on $\Omega_{\epsilon} \times \Omega$, plurisubharmonic in $z$, decreasing to $g(z, u)$ when $\epsilon \searrow 0$. Define, for $z \in \Omega_{\epsilon}$,

$$
p_{\mu, \epsilon}(z)=\int_{\Omega} g_{\epsilon}(z, u) d \mu(u) .
$$

Let $\chi \geqslant 0$ be a $C^{\infty}$ function with compact support in $\Omega$. Let $\epsilon>$ 0 be so small that supp $\chi \subset \Omega_{\epsilon}$. For brevity, we introduce the notation $M A\left(u_{1}, \ldots, u_{n}\right)$ for $d d_{z}^{c} u_{1} \wedge \ldots \wedge d d_{z}^{c} u_{n}$, where $u_{1}, \ldots, u_{n}$ are $C^{2}$ functions in $z$. Then we have, using (8) and Fubini's theorem repeatedly:

$$
\begin{aligned}
& \int_{z \in \Omega} \chi(z) \int_{\Omega^{n}} M A\left(g_{\epsilon}\left(z, v_{1}\right), \ldots, g_{\epsilon}\left(z, v_{n}\right)\right) d \mu\left(v_{1}\right) \cdots d \mu\left(v_{n}\right) \\
= & \int_{\Omega^{n}}\left(\int_{z \in \Omega} \chi(z) M A\left(g_{\epsilon}\left(z, v_{1}\right), \ldots g_{\epsilon}\left(z, v_{n}\right)\right)\right) d \mu\left(v_{1}\right) \cdots d \mu\left(v_{n}\right) \\
= & \int_{\Omega^{n}}\left(\int_{z \in \Omega} g_{\epsilon}\left(z, v_{1}\right) M A\left(\chi(z), g_{\epsilon}\left(z, v_{2}\right), \ldots, g_{\epsilon}\left(z, v_{n}\right)\right)\right) d \mu\left(v_{1}\right) \cdots d \mu\left(v_{n}\right) \\
= & \int_{z \in \Omega}\left(\int_{\Omega} g_{\epsilon}\left(z, v_{1}\right) d \mu\left(v_{1}\right)\right) \\
= & \int_{z \in \Omega} p_{\Omega^{n-1}} M A\left(\chi(z), g_{\epsilon}\left(z, v_{2}\right), \ldots, g_{\epsilon}\left(z, v_{n}\right)\right) d \mu\left(v_{2}\right) \cdots d \mu\left(v_{n}\right) \\
= & \left.\int_{\Omega^{n-1}}\left(\int_{z \in \Omega} p_{\mu, \epsilon}(z) M A\left(\chi(z), g_{\epsilon}\left(z, v_{2}\right), \ldots, g_{\epsilon}\left(z, v_{n}\right)\right) d \mu\left(v_{2}\right) \cdots d \mu\left(v_{n}\right), g_{\epsilon}\left(z, v_{2}\right), \ldots, g_{\epsilon}\left(z, v_{n}\right)\right)\right) d \mu\left(v_{2}\right) \cdots d \mu\left(v_{n}\right) \\
& =\int_{\Omega^{n-1}}\left(\int_{z \in \Omega} g_{\epsilon}\left(z, v_{2}\right) M A\left(\chi(z), p_{\mu, \epsilon}(z), g_{\epsilon}\left(z, v_{3}\right), \ldots, g_{\epsilon}\left(z, v_{n}\right)\right)\right) \\
& =\operatorname{etc.}=\int_{\Omega} \chi(z)\left(d d^{c} p_{\mu, \epsilon}(z)\right)^{n} \rightarrow \int_{\Omega} \chi(z)\left(d d^{c} p_{\mu}(z)\right)^{n},
\end{aligned}
$$


when $\epsilon \searrow 0$. In this computation all expressions of the types

$$
M A\left(\varphi_{1}, \ldots, \varphi_{n}\right)
$$

or

$$
\int_{\Omega} M A\left(\varphi_{1}, \ldots, \varphi_{n}\right) d \mu\left(v_{k}\right) \cdots d \mu\left(v_{n}\right)
$$

should be interpreted as measures in the variable $z$.

To complete the proof of (6) we must assure that the sequence of measures

$$
\int_{\Omega^{n}} d d_{z}^{c} g_{\epsilon}\left(z, v_{1}\right) \wedge \ldots \wedge d d_{z}^{c} g_{\epsilon}\left(z, v_{n}\right) d \mu\left(v_{1}\right) \cdots d \mu\left(v_{n}\right)
$$

tends to

$$
\int_{\Omega^{n}} d d_{z}^{c} g\left(z, v_{1}\right) \wedge \ldots \wedge d d_{z}^{c} g\left(z, v_{n}\right) d \mu\left(v_{1}\right) \cdots d \mu\left(v_{n}\right)
$$

weakly when $\epsilon \searrow 0$. We need to justify an application of Lebesgue's dominated convergence theorem in the integral

$$
\int_{\Omega^{n}}\left(\int_{\Omega} \chi(z) d d_{z}^{c} g_{\epsilon}\left(z, v_{1}\right) \wedge \ldots \wedge d d_{z}^{c} g_{\epsilon}\left(z, v_{n}\right)\right) d \mu\left(v_{1}\right) \cdots d \mu\left(v_{n}\right),
$$

where $\chi \in C_{0}^{\infty}(\Omega)$. Since $\mu$ is assumed to be finite, it is sufficient to prove that the measures $d d_{z}^{c} g_{\epsilon}\left(z, v_{1}\right) \wedge \ldots \wedge d d_{z}^{c} g_{\epsilon}\left(z, v_{n}\right)$ have locally uniformly bounded mass for small $\epsilon$, independent of $\left(v_{1}, \ldots, v_{n}\right) \in \Omega^{n}$. This is done in the technical Lemma 7.4 below.

We have proved Equation (6). To prove Equation (5), simply repeat the computation with $g(z, u)$ replaced with $\log |z-u|$. Then use Lemma 7.4.

Finally to show that Equation (4) holds, we repeat the same computation again. This time the counterpart to Lemma 7.4 is obvious.

Lemma 7.4. - Let $\Omega$ be a bounded, hyperconvex domain in $\mathbb{C}^{n}$. For each $v \in \Omega$, assume that $g_{\epsilon}(\cdot, v)$ is the usual regularization of $g(\cdot, v)$ and that $\log _{\epsilon}(\cdot, v)$ is the regularization of $\log |\cdot-v|$. The for each compact set $K$ in $\Omega$, there exists a constant $C=C(K)>0$ and an $\epsilon_{0}=\epsilon_{0}(K)>0$ such that

$$
\int_{K} d d_{z}^{c} g_{\epsilon}\left(z, v_{1}\right) \wedge \ldots \wedge d d_{z}^{c} g_{\epsilon}\left(z, v_{n}\right) \leqslant C
$$

and

$$
\int_{K} d d_{z}^{c} \log _{\epsilon}\left(z, v_{1}\right) \wedge \ldots \wedge d d_{z}^{c} \log _{\epsilon}\left(z, v_{n}\right) \leqslant C,
$$

for all $v_{1}, \ldots, v_{n} \in \Omega$ and all $\epsilon \leqslant \epsilon_{0}$. 
Proof. - Let $\Omega_{\epsilon}=\{z \in \Omega: \operatorname{dist}(z, \partial \Omega)>\epsilon\}$. Take an open set $\Omega^{\prime}$ such that $K \subset \Omega^{\prime} \subset \subset \Omega$. For an $n$-tuple $\left(v_{1}, \ldots, v_{n}\right) \in \Omega^{n}$, let us assume that exactly $k$ of the $v_{j}$ are contained in $\overline{\Omega^{\prime}}$, and that these are $v_{1}, \ldots, v_{k}$. We apply Inequality (7) on $\Omega^{\prime}$ to deduce that, for some compact set $L \subset \Omega^{\prime} \backslash K$, we have

$$
\begin{aligned}
& \int_{K} d d_{z}^{c} g_{\epsilon}\left(z, v_{1}\right) \wedge \ldots \wedge d d_{z}^{c} g_{\epsilon}\left(z, v_{n}\right) \\
& \leqslant C_{1}\left\|g_{\epsilon}\left(z, v_{k+1}\right)\right\|_{L} \cdots\left\|g_{\epsilon}\left(z, v_{n}\right)\right\|_{L} \\
& \quad \times \int_{K} d d_{z}^{c} g_{\epsilon}\left(z, v_{1}\right) \wedge \ldots \wedge d d_{z}^{c} g_{\epsilon}\left(z, v_{k}\right) \wedge \beta^{n-k},
\end{aligned}
$$

for all $\epsilon$ such that $\overline{\Omega^{\prime}} \subset \Omega_{\epsilon}$. We then apply Lemma 7.2 on $\Omega$, with $\overline{\Omega^{\prime}}$ as $K^{\prime}$ (see the remark following Lemma 7.2). This gives us a compact set $L^{\prime} \subset \Omega \backslash \overline{\Omega^{\prime}}$, such that

$$
\begin{aligned}
& \int_{K} d d_{z}^{c} g_{\epsilon}\left(z, v_{1}\right) \wedge \ldots \wedge d d_{z}^{c} g_{\epsilon}\left(z, v_{k}\right) \wedge \beta^{n-k} \\
& \quad \leqslant C_{2}\left\|g_{\epsilon}\left(z, v_{1}\right)\right\|_{L^{\prime}} \cdots\left\|g_{\epsilon}\left(z, v_{k}\right)\right\|_{L^{\prime}}\left\|\frac{|z|^{2}}{4}\right\|_{L^{\prime}}^{n-k} .
\end{aligned}
$$

Hence

$$
\begin{aligned}
& \int_{K} d d_{z}^{c} g_{\epsilon}\left(z, v_{1}\right) \wedge \ldots \wedge d d_{z}^{c} g_{\epsilon}\left(z, v_{n}\right) \\
& \leqslant C_{3} \prod_{j=1}^{k}\left\|g_{\epsilon}\left(z, v_{j}\right)\right\|_{L^{\prime}} \prod_{j=k+1}^{n}\left\|g_{\epsilon}\left(z, v_{j}\right)\right\|_{L} \\
& \quad \leqslant C_{3} \prod_{j=1}^{k}\left\|g\left(z, v_{j}\right)\right\|_{L^{\prime}} \prod_{j=k+1}^{n}\left\|g\left(z, v_{j}\right)\right\|_{L}
\end{aligned}
$$

Recall that $g(z, w) \geqslant \log |z-w|-\log R$, where $R>0$ is chosen such that $\Omega \subset B(w, R)$ for all $w \in \Omega$. This implies that $\left\|g\left(z, v_{j}\right)\right\|_{L^{\prime}} \leqslant \log R-\log$ $\operatorname{dist}\left(L^{\prime}, \partial \Omega^{\prime}\right), j=1, \ldots k$, and $\left\|g\left(z, v_{j}\right)\right\|_{L} \leqslant \log R-\log \operatorname{dist}\left(L, \partial \Omega^{\prime}\right), j=$ $k+1, \ldots, n$. Hence

$$
\int_{K} d d_{z}^{c} g_{\epsilon}\left(z, v_{1}\right) \wedge \ldots \wedge d d_{z}^{c} g_{\epsilon}\left(z, v_{n}\right) \leqslant C(k),
$$

where $C(k)$ is a constant which depends only on the number of poles inside $\Omega^{\prime}$. Define $C=\max _{0 \leqslant k \leqslant n} C(k)$. Then

$$
\int_{K} d d_{z}^{c} g_{\epsilon}\left(z, v_{1}\right) \wedge \ldots \wedge d d_{z}^{c} g_{\epsilon}\left(z, v_{n}\right) \leqslant C,
$$

and we are done. For the logarithm, the proof is similar. 


\section{Magnus Carlehed}

For the logarithmic potential, it is possible to get an explicit expression for the Monge-Ampère measure. We will use the notation $\operatorname{det}(a, b)=a_{1} b_{2}-$ $a_{2} b_{1}$, where $a$ and $b \in \mathbb{C}^{2}$.

Proposition 7.5. - If $v \neq w \in \mathbb{C}^{2}$, we have

$$
d d_{z}^{c} \log |z-v| \wedge d d_{z}^{c} \log |z-w|=4 \cdot \frac{|\operatorname{det}(z-v, z-w)|^{2}}{|z-v|^{4}|z-w|^{4}} d \lambda(z),
$$

and this measure is absolutely continuous with respect to the Lebesgue measure. Moreover, if $\mu$ is a finite positive measure with compact support in $\mathbb{C}^{2}$, and $l p_{\mu}$ is locally bounded, then

$$
\left(d d^{c} l p_{\mu}(z)\right)^{2}=4\left(\int_{\mathbb{C}^{2} \times \mathbb{C}^{2}} \frac{|\operatorname{det}(z-v, z-w)|^{2}}{|z-v|^{4}|z-w|^{4}} d \mu(v) d \mu(w)\right) d \lambda(z) .
$$

Proof. - We compute the first measure using the formula

$$
\begin{aligned}
d d^{c} f(z) & \wedge d d^{c} g(z) \\
= & 16\left(\frac{\partial^{2} f}{\partial z_{1} \partial \bar{z}_{1}} \frac{\partial^{2} g}{\partial z_{2} \partial \bar{z}_{2}}+\frac{\partial^{2} f}{\partial z_{2} \partial \bar{z}_{2}} \frac{\partial^{2} g}{\partial z_{1} \partial \bar{z}_{1}}\right) d \lambda(z) \\
& -16\left(\frac{\partial^{2} f}{\partial z_{1} \partial \bar{z}_{2}} \frac{\partial^{2} g}{\partial z_{2} \partial \bar{z}_{1}}+\frac{\partial^{2} f}{\partial z_{2} \partial \bar{z}_{1}} \frac{\partial^{2} g}{\partial z_{1} \partial \bar{z}_{2}}\right) d \lambda(z)
\end{aligned}
$$

and the fact that

$$
\frac{\partial^{2} \log |z|}{\partial z_{i} \partial \bar{z}_{j}}=\frac{1}{2}\left(\frac{-\bar{z}_{i} z_{j}}{|z|^{4}}+\frac{\delta_{i j}}{|z|^{2}}\right) .
$$

We omit the details. To prove the claim about absolute continuity, note that

$$
\frac{|\operatorname{det}(z-v, z-w)|^{2}}{|z-v|^{2}|z-w|^{2}}
$$

is uniformly bounded from above. Since $|z|^{-2} \in L_{\text {loc }}^{1}\left(\mathbb{C}^{2}\right)$, the claim is proved. Finally, inserting Equation (9) into Equation (5) gives Equation (10).

\section{Is there any relation between $\mu$ and $\left(d d^{c} p_{\mu}\right)^{n}$ ?}

In classical potential theory we have $\Delta U^{\mu}=c \mu$, where $U^{\mu}$ denotes the classical potential of the measure $\mu$ and $c$ is a positive constant. It is natural to ask whether there is any relation between the measures $\left(d d^{c} p_{\mu}\right)^{n}$ and $\mu$, such as an inequality. The following results partially answer this question. 
THEOREM 8.1. - Let $\Omega$ be a bounded, hyperconvex domain in $\mathbb{C}^{n}, n \geqslant 2$. Let $\mu$ be a finite measure, such that the logarithmic potential of $\mu$ is bounded. Then $\left(d d^{c} l p_{\mu}(z)\right)^{n}$ is an absolutely continuous measure with respect to the Lebesgue measure. If $\mu$ is such that the pluricomplex potential is bounded and $g(\cdot, v) \in C^{2}(\Omega \backslash\{v\})$ for all $v \in \Omega$, then $\left(d d^{c} p_{\mu}\right)^{n}$ is an absolutely continuous measure with respect to the Lebesgue measure.

Proof. - We prove the theorem for $p_{\mu}$, the proof for $l p_{\mu}$ being essentially the same. Let $K$ be a set of Lebesgue measure zero. Then using Equation (6) and Fubini's theorem we get

$$
\begin{aligned}
& \int_{K}\left(d d^{c} p_{\mu}(z)\right)^{n} \\
& \quad=\int_{K} \int_{\Omega^{n}} d d_{z}^{c} g\left(z, v_{1}\right) \wedge \ldots \wedge d d_{z}^{c} g\left(z, v_{n}\right) d \mu\left(v_{1}\right) \cdots d \mu\left(v_{n}\right) \\
& \quad=\int_{\Omega^{n}}\left(\int_{z \in K} d d_{z}^{c} g\left(z, v_{1}\right) \wedge \ldots \wedge d d_{z}^{c} g\left(z, v_{n}\right)\right) d \mu\left(v_{1}\right) \cdots d \mu\left(v_{n}\right) .
\end{aligned}
$$

Call the bracketed integral $f\left(v_{1}, \ldots, v_{n}\right)$. If $v_{i} \neq v_{j}$, for some indices $i$ and $j$, we have $f\left(v_{1}, \ldots, v_{n}\right)=0$, according to Proposition 4.4. If all the variables have the same value $v$, then $f(v, \ldots, v)=(2 \pi)^{n}$ if $v \in K$, with $f(v, \ldots, v)=0$ otherwise. Let $D=\{(v, \ldots, v): v \in \Omega\} \subset \Omega^{n}$. Then we have

$$
\begin{aligned}
\int_{K}\left(d d^{c} p(z)\right)^{n} & =\int_{\Omega^{n}} f\left(v_{1}, \ldots, v_{n}\right) d \mu\left(v_{1}\right) \cdots d \mu\left(v_{n}\right) \\
& \leqslant(2 \pi)^{n} \int_{D} d \mu\left(v_{1}\right) \cdots d \mu\left(v_{n}\right) \\
& =(2 \pi)^{n} \int_{\Omega}\left(\int_{\{w\}} d \mu(v)\right)^{n-1} d \mu(w) .
\end{aligned}
$$

Since $p$ is bounded, $\mu$ can not have any mass at points. Hence the inner integral is zero for all $w$, which implies $\int_{K}\left(d d^{c} p_{\mu}(z)\right)^{n}=0$, and the proposition is proved.

EXAMPLE 8.2. - Let $\Omega=B(0,1) \subseteq \mathbb{C}^{2}$, and let $\mu$ be the normalized Lebesgue measure on the disc $D(0,1) \times\{0\}$. Then the pluricomplex potential is bounded. Hence it cannot have any mass on the 1-dimensional complex variety $D(0,1) \times\{0\}$.

Proof. - From Proposition 3.1 we know that

$$
p_{\mu}(z)=\frac{\left(|z|^{2}-1\right)\left(1-\left|z_{2}\right|^{2}+\left|z_{2}\right|^{2} \log \left|z_{2}\right|^{2}\right)}{2\left(1-\left|z_{2}\right|^{2}\right)^{2}} .
$$


It is easy to see by using routine calculus that $-1 / 2=p_{\mu}(0) \leqslant p_{\mu}(z) \leqslant 0$. The second statement then follows from general properties of plurisubharmonic functions (see $[\mathrm{Kl}]$ ).

The moral here is that, when $n \geqslant 2$, the procedure $\mu \mapsto p_{\mu} \mapsto\left(d d^{c} p_{\mu}\right)^{n}$ is "smoothing". The fact that $\mu$ is singular is not visible at the end, as long as the singularity is not too strong. This breaks down in dimension 1 . We will generalize the result in Section 9.

Also note that Theorem 8.1 says that a necessary condition for a bounded plurisubharmonic function to be a pluricomplex potential is that its MongeAmpère measure is absolutely continuous with respect to the Lebesgue measure. Since there are many such functions whose Monge-Ampère measure is not absolutely continuous, this indicates that the Green functions (with one pole) form a very small subset of all extremal plurisubharmonic functions (see the discussion in Section 1).

THEOREM 8.3. - Let $\Omega$ be a bounded domain in $\mathbb{C}^{n}$. Let $q>1, f \in$ $L^{q}(\Omega)$ with $f \geqslant 0, d \mu=f d \lambda$. Then the pluricomplex and the logarithmic potentials of $\mu$ are continuous on $\bar{\Omega}$. Moreover, if $n=2$, and $f$ is not identically zero, then $\left(d d^{c} l p(z)\right)^{2}=\varphi(z) d \lambda(z)$, where $\varphi(z)>0$ throughout $\bar{\Omega}$.

Proof. - Since for each $z \in \Omega$ and $1 \leqslant r<\infty$, we have $\log |z-\cdot| \epsilon$ $L^{r}(\lambda, \Omega)$, it is clear that $l p_{\mu}(z)>-\infty$ for each $z$. Let $1 / q+1 / r=1$. We first prove that the logarithmic potential is continuous at every $z \in \Omega$. Without loss of generality, we may assume that $z=0$. Let $z_{j} \rightarrow 0$. Then

$$
\begin{aligned}
\mid l p_{\mu}\left(z_{j}\right) & -l p_{\mu}(0) \mid \\
\quad & \left|\int\left(\log \left|z_{j}-u\right|-\log |u|\right) f(u) d \lambda(u)\right| \\
& \leqslant\left(\int|\log | z_{j}-\left.u|-\log | u\right|^{r} d \lambda(u)\right)^{1 / r}\left(\int f(u)^{q} d \lambda(u)\right)^{1 / q},
\end{aligned}
$$

by Hölder's inequality. Hence it suffices to show that

$$
\int_{\Omega}|\log | z_{j}-u|-\log | u||^{r} d \lambda(u) \rightarrow 0 .
$$

This, however, follows immediately from Theorem 2.4.2 in [Ku-J-F], and the first statement is proved for $l p_{\mu}$.

We now turn to $p_{\mu}$. Again, it is enough to show continuity at $z=0$. If $\bar{B}\left(w, R_{1}\right) \subset \Omega \subset B\left(w, R_{2}\right)$, then $-\log R_{2} \leqslant g(z, w)-\log |z-w| \leqslant-\log R_{1}$, for all $z, w \in \Omega$. Hence, if $\delta>0$ is small enough, there is a constant $A$ such that $|g(z, w)-\log | z-w|| \leqslant A$, for all $z, w \in B(0, \delta)$. Let $\epsilon>0$ be given. 
Choose $\delta>0$, such that $A^{r} \cdot \operatorname{vol}(B(0, \delta))<\epsilon^{r}$. We have by Minkowski's inequality:

$$
\begin{aligned}
\left(\int_{B(0, \delta)}\left|g\left(z_{j}, u\right)-g(0, u)\right|^{r} d \lambda(u)\right)^{1 / r} & \\
\leqslant & \left(\int_{B(0, \delta)}\left|g\left(z_{j}, u\right)-\log \right| z_{j}-u||^{r} d \lambda(u)\right)^{1 / r} \\
& +\left(\int_{B(0, \delta)}|\log | z_{j}-\left.u|-\log | u\right|^{r} d \lambda(u)\right)^{1 / r} \\
& +\left(\left.\int_{B(0, \delta)}|g(0, u)-\log | u\right|^{r} d \lambda(u)\right)^{1 / r} \\
\leqslant & +\left(\int_{B(0, \delta)}|\log | z_{j}-\left.u|-\log | u\right|^{r} d \lambda(u)\right)^{1 / r}+\epsilon .
\end{aligned}
$$

Using the proof for $l p_{\mu}$, we conclude that $\int_{B(0, \delta)}\left|g\left(z_{j}, u\right)-g(0, u)\right|^{r} d \lambda(u)<$ $3 \epsilon$, if $j$ is large enough. Outside $B(0, \delta)$, all functions are uniformly bounded if $j$ is large enough. Hence we can use Lebesgue's dominated convergence theorem there, and the first statement is proved for $p_{\mu}$.

Finally, to prove the statement about $\varphi$, we use Equation (10):

$$
\varphi(z)=4 \int_{\Omega \times \Omega} \frac{|\operatorname{det}(z-v, z-w)|^{2}}{|z-v|^{4}|z-w|^{4}} f(v) f(w) d \lambda(v) d \lambda(w) .
$$

There is an $\epsilon>0$ and a set with positive Lebesgue measure where $f \geqslant \epsilon$. Since the determinant vanishes only in a set in $\Omega \times \Omega$ with Lebesgue measure zero, the integral is greater than zero for all $z$.

TheOREM 8.4. - Let $\Omega$ be a domain in $\mathbb{C}^{2}$. Let $f \geqslant 0, d \mu=f d \lambda$. Then there is a constant $C>0$ (depending only on $\Omega$ ), such that

$$
\left(d d^{c} l p_{\mu}(z)\right)^{2} \leqslant C\|f\|_{L^{q}}^{2}(2-r)^{-2 / r} d \lambda(z)
$$

for all $q>2, f \in L^{q}(\Omega)$ and $1 / q+1 / r=1$.

Proof. - It is clear that for all $z, v, w \in \Omega$,

$$
\begin{gathered}
\frac{|\operatorname{det}(z-v, z-w)|^{2}}{|z-v|^{2}|z-w|^{2}} \\
-457-
\end{gathered}
$$


is uniformly bounded. Hence, using Equation (10), we have

$$
\begin{aligned}
\left(d d^{c} l p_{\mu}(z)\right)^{2} & =4\left(\int_{\Omega \times \Omega} \frac{|\operatorname{det}(z-v, z-w)|^{2}}{|z-v|^{4}|z-w|^{4}} d \mu(v) d \mu(w)\right) d \lambda(z) \\
& \leqslant C_{1}\left(\int_{\Omega \times \Omega} \frac{f(v) f(w)}{|z-v|^{2}|z-w|^{2}} d \lambda(v) d \lambda(w)\right) d \lambda(z) \\
& =C_{1}\left(\int_{\Omega} \frac{f(v)}{|z-v|^{2}} d \lambda(v)\right)^{2} d \lambda(z) .
\end{aligned}
$$

Since $q>2$ we have $r<2$. Hence $|z-v|^{-2} \in L^{r}$ and the last integral can be estimated by Hölder's inequality:

$$
\int_{B} \frac{f(v)}{|z-v|^{2}} d \lambda(v) \leqslant\|f\|_{L^{q}}\left(\int_{\Omega}|z-v|^{-2 r} d \lambda(v)\right)^{1 / r} .
$$

Take $R>0$ large enough to ensure that $\Omega \subset B(z, R)$ for all $z \in \Omega$. Then we have

$$
\begin{aligned}
\int_{\Omega}|z-v|^{-2 r} d \lambda(v) & \left.\leqslant \int_{B(z, R)}|z-v|^{-2 r} d \lambda(v)=\text { s spherical coordinates }\right\} \\
& =C_{2} \int_{0}^{R} \rho^{3-2 r} d \rho=C_{2} \frac{R^{4-2 r}}{4-2 r}=C_{3}(2-r)^{-1} .
\end{aligned}
$$

Putting everything together we obtain the theorem.

We close this section with a characterization of smooth potentials.

THEOREM 8.5. - Let $\Omega$ be a domain in $\mathbb{C}^{n}$. Let $\mu$ be a measure (not necessarily bounded) in $\Omega$. Then the logarithmic potential is smooth (i.e. $\left.C^{\infty}\right)$ in $\Omega$ if and only if $\mu$ is given by a $C^{\infty}$ function times the Lebesgue measure. If $\Omega$ is such that $g(x, y)-\log |x-y| \in C^{\infty}(\Omega \times \Omega)$ then the same characterization holds for the pluricomplex potential. In particular, this is true for the unit ball.

Proof. - We begin with the logarithmic case. As mentioned in Section 2 , the logarithm is a fundamental solution of $\Delta^{n}$ in $\mathbb{R}^{2 n}$, and the potential is the convolution of the logarithm and the measure. The first statement of the theorem now follows from distribution theory, see [Hö], Theorem 4.4.1. The second statement is a trivial consequence of the first. For the ball, set $g(x, y)=\log |x-y|+h(x, y)$. We must prove that $h(x, y) \in C^{\infty}(B \times B)$. We omit the easy computation. 


\section{Measures whose potentials have finite energy}

Recently, Cegrell [Ce1] introduced the concept pluricomplex energy, and defined the Monge-Ampère operator on functions of finite energy.

Definition 9.1 [Ce1]. - Let $\Omega$ be a hyperconvex, bounded domain in $\mathbb{C}^{n}$. We define a function $u$ to be in the class $E_{0}$ if it is bounded, plurisubharmonic, tends to zero at the boundary, and $\int_{\Omega}\left(d d^{c} u\right)^{n}<\infty$. We define a function $u$ to be in the class $E_{p}$, if there is a sequence $\left\{u_{j}\right\} \subset E_{0}$, such that $u_{j} \searrow u$, and

$$
\sup _{j} \int\left(-u_{j}\right)^{p}\left(d d^{c} u_{j}\right)^{n}<\infty .
$$

It is shown that $E_{p}$ is a convex cone. We remark that functions with logarithmic poles, for instance the logarithm or the pluricomplex Green function, are not contained in $E_{p}$ for any $p$. We may say that functions in the class $E_{p}$ have finite p-energy. $E_{p}$.

THEOREM 9.2 [Ce1]. - The Monge-Ampère operator is well-defined on

The definition of the Monge-Ampère operator is done by means of the defining sequence, and it is shown that this gives a unique measure.

We begin with investigating what happens if we take a function $u \in E_{0}$, and perform the procedure

$$
u \mapsto\left(d d^{c} u\right)^{n} \mapsto p_{\left(d d^{c} u\right)^{n}} .
$$

Proposition 9.3. - Let $\Omega$ be a hyperconvex domain in $\mathbb{C}^{n}$, such that the pluricomplex Green function is symmetric, for example, a convex domain. Let $u \in E_{0}$ and set $\mu=\left(d d^{c} u\right)^{n}$. Then $p_{\mu} \in E_{0}$.

Proof. - We need to show that $p_{\mu}$ is bounded. Fix $z \in \Omega$, and set

$$
v^{N}(w)=\max \{g(w, z), N\} .
$$

Set $K=-\inf _{\zeta \in \Omega} u(\zeta)$. We have by Hölder's inequality

$$
\begin{gathered}
\int_{\Omega}-\max \{g(z, w), N\} d \mu(w) \\
=\int_{\Omega}-v^{N}(w)\left(d d^{c} u\right)^{n}(w) \\
\leqslant\left(\int_{\Omega}\left(d d^{c} u\right)^{n}\right)^{1-1 / n}\left(\int_{\Omega}\left(-v^{N}(w)\right)^{n}\left(d d^{c} u\right)^{n}(w)\right)^{1 / n} \\
-459-
\end{gathered}
$$




\section{Magnus Carlehed}

for $N \leqslant-1$. We now apply Corollary 2.2 in [Bł1] to obtain

$$
\begin{aligned}
\int_{\Omega}-\max \{g(z, w), N\} d \mu(w) & \leqslant\left(\int_{\Omega}\left(d d^{c} u\right)^{n}\right)^{1-1 / n}\left(n ! K^{n} \int_{\Omega}\left(d d^{c} v^{N}\right)^{n}\right)^{1 / n} \\
& \leqslant 2 \pi K(n !)^{1 / n}\left(\int_{\Omega}\left(d d^{c} u\right)^{n}\right)^{1-1 / n}
\end{aligned}
$$

Since

$$
-p_{\mu}(z)=\lim _{N \rightarrow-\infty} \int_{\Omega}-\max \{g(z, w), N\} d \mu(w),
$$

it follows that $p_{\mu}$ is bounded. We also need to check that $\left(d d^{c} p_{\mu}\right)^{n}$ is finite. We use Equation (6):

$$
\int_{\Omega}\left(d d^{c} p_{\mu}\right)^{n}=\int_{\Omega^{n}}\left(\int_{z \in \Omega} d d_{z}^{c} g\left(z, v_{1}\right) \wedge \ldots \wedge d d_{z}^{c} g\left(z, v_{n}\right)\right) d \mu\left(v_{1}\right) \cdots d \mu\left(v_{n}\right) .
$$

Recall that the inner integral is uniformly bounded in $v_{1}, \ldots, v_{n}$. Hence

$$
\int_{\Omega}\left(d d^{c} p_{\mu}\right)^{n} \leqslant C\left(\int d \mu\right)^{n}
$$

and $\int d \mu=\int\left(d d^{c} u\right)^{n}<\infty$ by assumption. The proof is complete.

In the rest of this section we will work in the unit ball in $\mathbb{C}^{2}$ and prove that $E_{1}$ contains the potential of a measure $\mu$, if $\mu$ satisfies a kind of "logarithmic finite energy" condition.

THEOREM 9.4. - Let $\Omega=B$, the unit ball in $\mathbb{C}^{2}$. If

$$
\int_{B \times B} \frac{-\log |x-y|}{(1-|x|)(1-|y|)} d \mu(x) d \mu(y)<\infty,
$$

then $p_{\mu} \in E_{1}$, and

$$
\left(d d^{c} p_{\mu}\right)^{2}(z)=\int_{B \times B} d d_{z}^{c} g(z, v) \wedge d d_{z}^{c} g(z, w) d \mu(v) d \mu(w) .
$$

Furthermore, $\left(d d^{c} p_{\mu}\right)^{2}$ is an absolutely continuous measure with respect to the Lebesgue measure.

$$
\begin{aligned}
& \text { Proof. - Let } \\
& \qquad g_{N}(z, w)=\max \{g(z, w), N\},
\end{aligned}
$$

and define, for $N \leqslant-1$,

$$
\begin{aligned}
p_{N}(z, w)= & \int_{B} g_{N}(z, w) d \mu(w) \\
& -460-
\end{aligned}
$$




\section{Potentials in pluripotential theory}

Then $p_{N}$ is a bounded function on $\Omega \times \Omega$, plurisubharmonic in $z$, so we can apply Equation (4) to obtain

$$
\left(d d^{c} p_{N}\right)^{2}(z)=\int_{B \times B} d d_{z}^{c} g_{N}(z, v) \wedge d d_{z}^{c} g_{N}(z, w) d \mu(v) d \mu(w) .
$$

Since, by Corollary 5.4 , the total mass of the measures

$$
d d_{z}^{c} g_{N}(z, v) \wedge d d_{z}^{c} g_{N}(z, w)
$$

is bounded (uniformly in $v, w$ and $N$ ), we have

$$
\int_{B}\left(d d^{c} p_{N}\right)^{2}=\int_{B \times B}\left(\int_{B} d d_{z}^{c} g_{N}(z, v) \wedge d d_{z}^{c} g_{N}(z, w)\right) d \mu(v) d \mu(w)<\infty .
$$

It follows that that each $p_{N}$ is in $E_{0}$. Since also $p_{N} \searrow p_{\mu}$ when $N \rightarrow-\infty$, it is sufficient to show that if Inequality (12) holds, then $\sup _{N} \int_{B}-p_{N}\left(d d^{c} p_{N}\right)^{2}<$ $\infty$. We have

$$
\begin{aligned}
\int_{B} & -p_{N}\left(d d^{c} p_{N}\right)^{2} \\
& =\int_{B \times B \times B}\left(\int_{z \in B}-g_{N}(z, t) d d_{z}^{c} g_{N}(z, v) \wedge d d_{z}^{c} g_{N}(z, w)\right) d \mu(t) d \mu(v) d \mu(w) .
\end{aligned}
$$

Let $I(t, v, w)$ be the inner integral here. We can estimate it using Theorem 5.5 in the following way.

$$
\begin{aligned}
I(t, v, w) & =\int_{z \in B}-g(z, t) d d_{z}^{c} g_{N}(z, v) \wedge d d_{z}^{c} g_{N}(z, w) \\
& =\int-g_{N}(z, v) d d_{z}^{c} g(z, t) \wedge d d_{z}^{c} g_{N}(z, w) \\
& \leqslant \int-g(z, v) d d_{z}^{c} g(z, t) \wedge d d_{z}^{c} g_{N}(z, w) \\
& =\int-g_{N}(z, w) d d_{z}^{c} g(z, t) \wedge d d_{z}^{c} g(z, v) \\
& \leqslant \int-g(z, w) d d_{z}^{c} g(z, t) \wedge d d_{z}^{c} g(z, v) \\
& =\int-g(z, t) d d_{z}^{c} g(z, v) \wedge d d_{z}^{c} g(z, w) .
\end{aligned}
$$

We claim that if $v \neq w$, then

$$
\begin{gathered}
d d_{z}^{c} g(z, v) \wedge d d_{z}^{c} g(z, w) \leqslant \frac{4}{(1-|v|)(1-|w|)} \cdot \frac{1-\mid\left\langle\frac{z-v}{|z-v|},|z-w|\right.}{\left.|z-v|\right|^{2}|z-w|^{2}} d \lambda(z) . \\
-461-
\end{gathered}
$$


This estimate is contained in Lemma 9.6 below, and combining it with a trivial estimate of $g$, we conclude that

$$
I(t, v, w) \leqslant \frac{4}{(1-|v|)(1-|w|)} \int_{B}-\log \frac{|z-t|}{4} \cdot \frac{1-\left|\left\langle\frac{z-v}{|z-v|}, \frac{z-w}{|z-w|}\right\rangle\right|^{2}}{|z-v|^{2}|z-w|^{2}} d \lambda(z) .
$$

Since we know that

$$
\int_{B \times B} \frac{A-B \log |x-y|}{(1-|x|)(1-|y|)} d \mu(x) d \mu(y)<\infty
$$

for every $A, B \geqslant 0$ by assumption, it suffices to prove that there exists $A, B \geqslant 0$ such that

$$
\int_{B}-\log \frac{|z-t|}{4} \cdot \frac{1-\left|\left\langle\frac{z-v}{|z-v|}, \frac{z-w}{|z-w|}\right\rangle\right|^{2}}{|z-v|^{2}|z-w|} d \lambda(z) \leqslant A-B \log |v-w| .
$$

This is done in the technical Lemma 9.8 below.

Next we prove Formula (13). By Theorem 9.2 and its proof, $\left(d d^{c} p_{\mu}\right)^{2}$ is well-defined and equals the weak limit of $\left(d d^{c} p_{N}\right)^{2}$. Hence it suffices to show that if $0 \leqslant \chi(z) \in C_{0}^{\infty}(B)$, then

$$
\begin{aligned}
J & :=\int_{z \in B} \chi(z) \int_{B^{2}} d d_{z}^{c} g_{N}(z, v) \wedge d d_{z}^{c} g_{N}(z, w) d \mu(v) d \mu(w) \\
& \rightarrow \int_{z \in B} \chi(z) \int_{B^{2}} d d_{z}^{c} g(z, v) \wedge d d_{z}^{c} g(z, w) d \mu(v) d \mu(w) .
\end{aligned}
$$

But

$$
J=\int_{B^{2}}\left(\int_{z \in B} \chi(z) d d_{z}^{c} g_{N}(z, v) \wedge d d_{z}^{c} g_{N}(z, w)\right) d \mu(v) d \mu(w),
$$

and the inner integral here is bounded by

$$
\|\chi\|_{L^{\infty}} \int_{B} d d_{z}^{c} g_{N}(z, v) \wedge d d_{z}^{c} g_{N}(z, w)
$$

which, again, is uniformly bounded in $v, w$ and $N$. Hence we can apply Lebesgue's dominated convergence theorem, and Formula (13) is proved.

Finally, to prove the claim about absolute continuity, repeat the proof of Theorem 8.1. This is possible since a measure satisfying Condition (12) can not have any mass at points.

It remains to show a number of technical lemmas, used in the preceding proof. The first one is a straight-forward but tedious computation, and we omit the details. 
LEMMA 9.5. - We have if $u \neq v$,

$$
\begin{aligned}
d d_{z}^{c} g(z, u) \wedge d d_{z}^{c} g(z, v) & \frac{4\left(1-|u|^{2}\right)\left(1-|v|^{2}\right)|\operatorname{det}(z-u, z-v)|^{2}}{\left(|z-u|^{2}-|\operatorname{det}(z-u, u)|^{2}\right)^{2}\left(|z-v|^{2}-|\operatorname{det}(z-v, v)|^{2}\right)^{2}} d \lambda(z) \\
= & \frac{1-\left|\left\langle\frac{z-u}{|z-u|}, \frac{z-v}{|z-v|}\right\rangle\right|^{2}}{|z-u|^{2}|z-v|^{2}} \\
& \times \frac{4\left(1-|u|^{2}\right)\left(1-|v|^{2}\right)}{\left[1-|u|^{2}+\left|\left\langle\frac{z-u}{|z-u|}, u\right\rangle\right|^{2}\right]^{2}\left[1-|v|^{2}+\left|\left\langle\frac{z-v}{|z-v|}, v\right\rangle\right|^{2}\right]^{2}} d \lambda(z) .
\end{aligned}
$$

LEMMA 9.6. - We have if $u \neq v$,

$$
d d_{z}^{c} g(z, u) \wedge d d_{z}^{c} g(z, v) \leqslant \frac{1-\left|\left\langle\frac{z-u}{|z-u|}, \frac{z-v}{|z-v|}\right\rangle\right|^{2}}{|z-u|^{2}|z-v|^{2}} \times \frac{4}{(1-|u|)(1-|v|)} d \lambda(z) .
$$

Proof. - Immediate from the previous lemma.

Lemma 9.7. - Let $B_{2}=B(0,2) \subset \mathbb{C}^{2}$. Then the function $\Phi: \mathbb{C}^{2} \times \mathbb{C}^{2} \rightarrow$ $\mathbb{R}$, defined by

$$
\Phi(t, w)=\int_{B_{2}} \frac{\log \frac{|z||z-w|}{2|z-t|}}{|z|^{2}|z-w|^{2}} d \lambda(z)
$$

is bounded from above.

Proof. - Define $f_{c}(x)=\log (x / c) / x^{2}$ for $x \geqslant c>0$. By differentiation we see that $f_{c}(x) \leqslant 1 /\left(2 e c^{2}\right)$. Hence

$$
\begin{aligned}
\Phi(t, w) & \leqslant \int_{B_{2} \cap\{|z||z-w| \geqslant 2|z-t|\}} \frac{\log \frac{|z||z-w|}{2|z-t|}}{|z|^{2}|z-w|^{2}} d \lambda(z) \\
& \leqslant \frac{1}{8 e} \int_{B_{2}} \frac{d \lambda(z)}{|z-t|^{2}} \leqslant C,
\end{aligned}
$$

which proves the lemma.

LEMMA 9.8. - Let $B_{r}=B(0, r) \subset \mathbb{C}^{2}$, and define a function $\Psi: B_{1} \times$ $B_{1} \times B_{1} \rightarrow \mathbb{R}$ by

$$
\begin{gathered}
\Psi(t, u, v)=\int_{B_{1}} \frac{-\log \frac{|z-t|}{4} \cdot\left(1-\left|\left\langle\frac{z-u}{|z-u|}, \frac{z-v}{|z-v|}\right\rangle\right|^{2}\right)}{|z-u|^{2}|z-v|^{2}} d \lambda(z) . \\
-463-
\end{gathered}
$$




\section{Magnus Carlehed}

Then there are constants $A$ and $B>0$, such that

$$
\Psi(t, u, v) \leqslant A-B \log |u-v| .
$$

Proof. - Let $w=v-u$, and make the change of variables $z^{\prime}=z-u$, to obtain

$$
\Psi(t, u, v)=\int_{B(-u, 1)} \frac{-\log \frac{\left|z^{\prime}+u-t\right|}{4} \cdot\left(1-\left|\left\langle\frac{z^{\prime}}{\left|z^{\prime}\right|}, \frac{z^{\prime}-w}{\left|z^{\prime}-w\right|}\right\rangle\right|^{2}\right)}{\left|z^{\prime}\right|^{2}\left|z^{\prime}-w\right|^{2}} d \lambda\left(z^{\prime}\right) .
$$

Since $B(-u, 1) \subset B(0,2)$ and the integrand is non-negative for $\left|z^{\prime}\right|<2$, we get

$$
\Psi(t, u, v) \leqslant \int_{B_{2}} \frac{-\log \frac{|z+u-t|}{4} \cdot\left(1-\left|\left\langle\frac{z}{|z|}, \frac{z-w}{|z-w|}\right\rangle\right|^{2}\right)}{|z|^{2}|z-w|^{2}} d \lambda(z) .
$$

We split this into three integrals $I_{1}, I_{2}$ and $I_{3}$, where

$$
\begin{gathered}
I_{1}=\int_{B_{2}} \frac{\log \frac{|z||z-w|}{2|z+u-t|} \cdot\left(1-\left|\left\langle\frac{z}{|z|}, \frac{z-w}{|z-w|}\right\rangle\right|^{2}\right)}{|z|^{2}|z-w|^{2}} d \lambda(z), \\
I_{2}=\int_{B_{2}} \frac{-\log \frac{|z|}{2} \cdot\left(1-\left|\left\langle\frac{z}{|z|}, \frac{z-w}{|z-w|}\right\rangle\right|^{2}\right)}{|z|^{2}|z-w|^{2}} d \lambda(z), \\
I_{3}=\int_{B_{2}} \frac{-\log \frac{|z-w|}{4} \cdot\left(1-\left|\left\langle\frac{z}{|z|}, \frac{z-w}{|z-w|}\right\rangle\right|^{2}\right)}{|z|^{2}|z-w|^{2}} d \lambda(z) .
\end{gathered}
$$

Note that

$$
0 \leqslant 1-\left|\left\langle\frac{z}{|z|}, \frac{z-w}{|z-w|}\right\rangle\right|^{2} \leqslant 1 .
$$

Using this and Lemma 9.7 we conclude that $I_{1}$ is bounded from above by $A_{1}$, say. To estimate $I_{2}$, note that it is a rotation invariant function of $w$. 
Potentials in pluripotential theory

Hence we may assume that $w=(w, 0)$ where $w \in \mathbb{R}^{+}$. We obtain

$$
\begin{aligned}
I_{2}= & \int_{B_{2}} \frac{-\log \frac{|z|}{2} \cdot|z|^{2}\left(|z|^{2}+w^{2}-2 w \operatorname{Re} z_{1}\right)}{|z|^{4}\left[|z|^{2}+w^{2}-2 w \operatorname{Re} z_{1}\right]^{2}} d \lambda(z) \\
& -\int_{B_{2}} \frac{-\log \frac{|z|}{2} \cdot\left(|z|^{4}+w^{2}\left|z_{1}\right|^{2}-2|z|^{2} w \operatorname{Re} z_{1}\right)}{|z|^{4}\left[|z|^{2}+w^{2}-2 w \operatorname{Re} z_{1}\right]^{2}} d \lambda(z) \\
= & w^{2} \int_{B_{2}} \frac{-\left|z_{2}\right|^{2} \log \frac{|z|}{2}}{|z|^{4}\left[|z|^{2}+w^{2}-2 w \operatorname{Re} z_{1}\right]^{2}} d \lambda(z) .
\end{aligned}
$$

We now change to spherical coordinates in $\mathbb{R}^{4}$. This gives

$$
I_{2}=2 \pi w^{2} \int_{0}^{\pi} \sin ^{3} \theta_{2} d \theta_{2} \cdot \int_{0}^{2}-r \log \frac{r}{2}\left(\int_{0}^{\pi} \frac{\sin ^{4} \theta_{1} d \theta_{1}}{\left(r^{2}+w^{2}-2 r w \cos \theta_{1}\right)^{2}}\right) d r .
$$

Call the bracketed integral $I_{4}$. To estimate $I_{4}$, we make the substitution $t=\tan \theta_{1} / 2$, and get

$$
\begin{aligned}
I_{4} & =\int_{0}^{\infty} \frac{\left(\frac{2 t}{1+t^{2}}\right)^{4}}{\left(r^{2}+w^{2}-2 r w \frac{1-t^{2}}{1+t^{2}}\right)^{2}} \cdot \frac{2 d t}{1+t^{2}} \\
& =32 \int_{0}^{\infty} \frac{t^{4} d t}{\left((r-w)^{2}+(r+w)^{2} t^{2}\right)^{2}\left(1+t^{2}\right)^{3}} \\
& \leqslant 32(r+w)^{-4} \int_{0}^{\infty} \frac{d t}{\left(1+t^{2}\right)^{3}}=C(r+w)^{-4} .
\end{aligned}
$$

Thus

$$
I_{2} \leqslant C w^{2} \int_{0}^{2} \frac{-r \log \frac{r}{2}}{(r+w)^{4}} d r \leqslant A_{2}-B_{2} \log w
$$

by direct integration. We conclude that $I_{2} \leqslant A_{2}-B_{2} \log |v-u|$.

For $I_{3}$, just note that since $|w|<2$,

$$
\begin{gathered}
I_{3}=\int_{B(-w, 2)} \frac{-\log \frac{\left|z^{\prime}\right|}{4} \cdot\left(1-\left|\left\langle\frac{z^{\prime}+w}{\left|z^{\prime}+w\right|}, \frac{z^{\prime}}{\left|z^{\prime}\right|}\right\rangle\right|^{2}\right)}{\left|z^{\prime}+w\right|^{2}\left|z^{\prime}\right|^{2}} d \lambda\left(z^{\prime}\right) \\
\leqslant \int_{B_{4}} \frac{-\log \frac{|z|}{4} \cdot\left(1-\left|\left\langle\frac{z+w}{|z+w|}, \frac{z}{|z|}\right\rangle\right|^{2}\right)}{|z+w|^{2}|z|^{2}} d \lambda(z) \\
-465-
\end{gathered}
$$


and we can estimate it exactly as $I_{2}$. We obtain $I_{3} \leqslant A_{3}-B_{3} \log |v-u|$. Putting everything together, we obtain $\Psi(t, u, v) \leqslant A_{1}+A_{2}-B_{2} \log |v-u|+$ $A_{3}-B_{3} \log |v-u|=A-B \log |v-u|$, as desired.

We remark that for measures $\mu$ with compact support, Condition (12) reduces to

$$
\int_{B \times B}-\log |x-y| d \mu(x) d \mu(y)<\infty .
$$

It would be interesting to see whether this condition is also necessary.

EXAMPLE 9.9. - In the unit ball in $\mathbb{C}^{2}$ we construct a measure whose potential is unbounded, but still in $E_{1}$.

Proof. - We adopt the convention that $C, C_{1}, C_{2}$ etc. are positive constants but they may have different values in different expressions. For example, we might write $C|f(x)| \leqslant C$, if we know that $|f(x)|$ is bounded by some other constant (not necessarily 1). Let $\left\{a_{j}\right\},\left\{b_{j}\right\} \subset(0,1)$ be sequences which decrease to zero. Further assume that $\sum a_{j} / b_{j}<\infty$, and $\sum a_{j} / b_{j}^{2}=\infty$. We will use radial measures of the type $d \mu=f(|x|) d \lambda(x)$, where $f(r)=\sum a_{j} r^{b_{j}-4}$ if $r<1 / 2$, and zero if $r \geqslant 1 / 2$. We first note that $\mu$ is finite, since

$$
\int d \mu=C \sum a_{j} \int_{0}^{1 / 2} r^{b_{j}-4} r^{3} d r=C \sum a_{j} / b_{j}<\infty .
$$

Further, $p_{\mu}$ is unbounded, since

$$
\begin{aligned}
p_{\mu}(0) & =\int \log |x| d \mu(x)=C \sum a_{j} \int_{0}^{1 / 2}(\log r) r^{b_{j}-4} r^{3} d r \\
& =-C \sum \frac{a_{j} 2^{-b_{j}}}{b_{j}}-C \sum \frac{a_{j} 2^{-b_{j}}}{b_{j}^{2}}=-\infty
\end{aligned}
$$

It remains to show that $\mu$ satisfies Condition (14). First note that since $\mu$ is rotation invariant, then so is $l p_{\mu}$. Let $x=(t, 0), t \in(0,1 / 2)$, and change to spherical coordinates. We get

$$
-l p_{\mu}(x)=C \sum a_{j} \int_{0}^{1 / 2}\left(\int_{0}^{\pi}-\log \left(t^{2}+r^{2}-2 r t \cos \theta\right) \sin ^{2} \theta d \theta\right) r^{-1+b_{j}} d r .
$$

Let $I$ be the inner integral here. We use the substitution $x=\tan \theta / 2$. This 
Potentials in pluripotential theory

gives

$$
\begin{aligned}
I & =\int_{0}^{\infty}-\log \left(t^{2}+r^{2}-2 r t \frac{1-x^{2}}{1+x^{2}}\right)\left(\frac{2 x}{1+x^{2}}\right)^{2} \frac{2 d x}{1+x^{2}} \\
& =-8 \int_{0}^{\infty} \log \left(\frac{(t-r)^{2}+(t+r)^{2} x^{2}}{1+x^{2}}\right) \frac{x^{2}}{\left(1+x^{2}\right)^{3}} d x \\
& =8 \int_{0}^{\infty} \log \left(1+x^{2}\right) \frac{x^{2}}{\left(1+x^{2}\right)^{3}} d x-16 \log |t-r| \int_{0}^{\infty} \frac{x^{2}}{\left(1+x^{2}\right)^{3}} d x \\
& -8 \int_{0}^{\infty} \log \left(1+\frac{(t+r)^{2} x^{2}}{(t-r)^{2}}\right) \frac{x^{2}}{\left(1+x^{2}\right)^{3}} d x \\
& =8 f(1)-16 \log |t-r| \int_{0}^{\infty} \frac{x^{2}}{\left(1+x^{2}\right)^{3}} d x-8 f\left(\frac{(t+r)^{2}}{(t-r)^{2}}\right),
\end{aligned}
$$

where $f$ is defined by

$$
f(a)=\int_{0}^{\infty} \log \left(1+a x^{2}\right) \frac{x^{2}}{\left(1+x^{2}\right)^{3}} d x .
$$

We have $f(0)=0$ and

$$
f^{\prime}(a)=\int_{0}^{\infty} \frac{x^{4}}{\left(1+a x^{2}\right)\left(1+x^{2}\right)^{3}} d x=\frac{\pi(3+\sqrt{a})}{16(1+\sqrt{a})^{3}},
$$

by elementary methods from calculus. Hence

$$
\begin{aligned}
f(a) & =f(0)+\int_{0}^{a} f^{\prime}(\alpha) d \alpha=\frac{\pi}{16} \int_{0}^{a} \frac{3+\sqrt{\alpha}}{(1+\sqrt{\alpha})^{3}} d \alpha \\
& =\frac{\pi}{8}\left(\log (1+\sqrt{a})-\frac{\sqrt{a}}{(1+\sqrt{a})^{2}}\right) .
\end{aligned}
$$

We also have

$$
\int_{0}^{\infty} \frac{x^{2}}{\left(1+x^{2}\right)^{3}} d x=\frac{\pi}{16}
$$

We conclude that

$$
\begin{aligned}
& \frac{I}{\pi}= \log 2-\frac{1}{4}-\log |t-r|-\log \left(1+\frac{r+t}{|t-r|}\right)+\frac{(r+t) /|t-r|}{(1+(r+t) /|t-r|)^{2}} \\
&=\log 2-\frac{1}{4}-\log (|t-r|+r+t)+\frac{\left|r^{2}-t^{2}\right|}{(|t-r|+r+t)^{2}} \\
&=-\frac{1}{4}-\log \max \{r, t\}+\frac{\left|r^{2}-t^{2}\right|}{4 \max \left\{r^{2}, t^{2}\right\}} \\
&-467-
\end{aligned}
$$


Thus

$$
\begin{gathered}
\frac{1}{\pi} \int_{0}^{1 / 2}\left(\int_{0}^{\pi}-\log \left(t^{2}+r^{2}-2 r t \cos \theta\right) \sin ^{2} \theta d \theta\right) r^{-1+b} d r \\
=\int_{0}^{t}\left(-\frac{1}{4}-\log t+\frac{t^{2}-r^{2}}{4 t^{2}}\right) r^{-1+b} d r \\
+\int_{t}^{1 / 2}\left(-\frac{1}{4}-\log r+\frac{r^{2}-t^{2}}{4 r^{2}}\right) r^{-1+b} d r \\
=\frac{2^{-b}(2+b) t^{2}-t^{b}}{4-b^{2}}+\frac{2^{-b} \log 2}{b}+\frac{2^{-b}-t^{b}}{b^{2}} \\
\leqslant C_{1}+\frac{C_{2}}{b}+C_{3} \frac{2^{-b}-t^{b}}{b^{2}}
\end{gathered}
$$

It follows that

$$
-l p_{\mu}(x) \leqslant \sum a_{j}\left(C_{1}+\frac{C_{2}}{b_{j}}+C_{3} \frac{2^{-b_{j}}-t^{b_{j}}}{b_{j}^{2}}\right) .
$$

Note that the last sum is convergent for all $0<t<1 / 2$, since $0<2^{-b}-t^{b} \leqslant$ $-b \log t$, by the Mean Value Theorem applied to the function $b \mapsto 2^{-b}-t^{b}$.

Now we integrate again, and obtain

$$
\begin{aligned}
\int-\log \mid & x-y \mid d \mu(x) d \mu(y) \\
& =\int-l p_{\mu}(x) d \mu(x) \\
& \leqslant C \sum a_{j} a_{k} \int_{0}^{1 / 2}\left(C_{1}+\frac{C_{2}}{b_{j}}+C_{3} \frac{2^{-b_{j}}-t^{b_{j}}}{b_{j}^{2}}\right) t^{-1+b_{k}} d t \\
& =\sum a_{j} a_{k}\left(\frac{C_{1}}{b_{k}}+\frac{C_{2}}{b_{j} b_{k}}+\frac{C_{3}}{b_{j}^{2}}\left(\frac{1}{b_{k}}-\frac{1}{b_{j}+b_{k}}\right)\right) \\
& =\sum a_{j} a_{k}\left(\frac{C_{1}}{b_{k}}+\frac{C_{2}}{b_{j} b_{k}}+\frac{C_{3}}{b_{j} b_{k}\left(b_{j}+b_{k}\right)}\right) .
\end{aligned}
$$

If we now choose, for instance, $a_{j}=j^{-3}, b_{j}=j^{-1}$, the last series converges, and the corresponding measure has finite energy.

Remark. - For the suggested choice of $a_{j}$ and $b_{j}$, the last series becomes $C_{1} \zeta(2) \zeta(3)+C_{2} \zeta(2)^{2}+2 C_{3} \zeta(3)$. Here we used the curious fact that

$$
\sum_{j, k=1}^{\infty} \frac{1}{j k(j+k)}=2 \zeta(3)
$$

which can be proved using an integral representation of the Riemann zeta function. 
Potentials in pluripotential theory

\section{Bibliography}

[A-Cr-Li] Aronszajn, N., Creese, T., Lipkin, L., Polyharmonic functions, Clarendon Press, Oxford 1983.

[Be-T] BEDFORD, E. and TAYLOR, B.A., A new capacity for plurisubharmonic functions, Acta Math. 149 (1982), 1-40.

[Bł1] BŁockI, Z. Estimates for the complex Monge-Ampère operator, Bull. Pol. Acad. Sci., Mathematics, 41, no 2 (1993), 151-157.

[B12] BŁockI, Z., The complex Monge-Ampère operator in pluripotential theory, lecture notes.

[Ca] CARLEHED, M., The pluricomplex Green function and related topics in pluripotential theory, Doctoral thesis No 12, 1998, Umeå University, Department of Mathematics.

[Ce1] Cegrell, U., Pluricomplex energy, Acta Math. 180:2 (1998), 187-217.

[Ce2] CEgrell, U., The symmetric pluricomplex Green function, Topics in complex analysis, Banach Center Publ. 31, Institute of Mathematics, Polish academy of sciences, Warsaw 1995.

[Ce3] Cegrell, U., Capacities in complex analysis, Vieweg, 1988.

[Ce4] Cegrell, U., Approximation of plurisubharmonic functions and integration by parts, report no 8,1998 , Mid Sweden University.

[Ce-T] Cegrell, U., ThorbiöRnson, J., Extremal plurisubharmonic functions, Ann. Pol. Math. 63:1 (1996), 63-69.

[D] Demailly, J.-P., Mesures de Monge-Ampère et mesures pluriharmoniques, Math. Z. 194 (1987), 519-564.

[Ha-Ke] Hayman, W.K., Kennedy, P.B., Subharmonic Functions, vol I, Academic Press, 1976.

[Hö] HöRMANDER, L., The analysis of linear partial differential operators I, Springer, 1991

[Kl] KLIMEK, M., Pluripotential Theory, Clarendon Press, 1991.

[Ku-J-F] KufNer, A., John, O., Fučík, S., Function spaces, Noordhoff, Leyden 1977.

[Lel1] LELONG, P., Fonctionnelles analytiques et fonctions entières ( $n$ variables), Les presses de l'université de Montréal, Montreal 1968.

[Lel2] Lelong, P., Fonction de Green pluricomplexe et lemmes de Schwarz dans les espaces de Banach, J. Math. Pures Appl. 68 (1989), 319-347.

[Lem] LEMPERT, L., Solving the degenerate complex Monge-Ampère equation with one concentrated singularity, Math. Ann. 263, no 4 (1983), 515-532.

[S] Sibony, N., Quelques problèmes de prolongement de courants en analyse complexe, Duke Math. J. 52 (1985), 157-197. 